\title{
Optimization Design of PID Controller for Middle and High Frequency Ranges
}

\author{
Yong Qiao \\ School of IoT Engineering \\ Jiangnan University \\ Wuxi 214122, China \\ qy_19900409@163.com
}

\author{
Xuyang Lou \\ School of IoT Engineering \\ Jiangnan University \\ Wuxi 214122, China \\ Lou047@gmail.com
}

\begin{abstract}
This paper presents a method of designing PID controller for both middle frequency range with typical signals input and high frequency range with ramp input. Based on the principle of approximate model matching, the design of PID controller can be converted into the problem of calculating optimal solution of a particular norm with the form of inequality for restricted frequency ranges. With the help of Kalman-Yakubovic-Popov (KYP) lemma, the frequency domain inequalities are transformed to linear matrix inequalities (LMIs) in the state space realization. Finally, a simulation example is provided to illustrate the effectiveness of the main results.
\end{abstract}

Keywords-middle and high frequencies; approximate model matching; bounded-realness theorem; PID controller

\section{INTRODUCTION}

As is well-known, $H_{\infty}$ norm is one kind of performance index in the entire frequency domain, which is established by Zames [1] and Dolye et al. [2]. In 2005, Iwasaki and Hara [3] stated that a certain frequency range rather than the entire frequency range is usually requested in practical design. As a consequence, the standard $H_{\infty}$ norm and the generalized bounded-realness theorem are not well compatible with practical requirements.

Recently, Iwasaki et al. [4] presented the generalization of Kalman-Yakubovic-Popov (KYP) lemma for restricted frequency inequalities. Specifically, they considered the frequency intervals characterized by two quadratic forms, which encompassed low/middle/high frequency conditions for continuous-time systems. Based on their results, Ma et al. gave out certain forms of bounded-realness theorem and its dual version for low frequency range (LFR). However, to best of the author's knowledge, results on the design of PID controller for both middle frequency range (MFR) with typical signals input and high frequency range (HFR) with ramp input do not seem available in the literature.

In this paper, according to the method of approximate matching model [6], we convert the optimization design of PID controller into the optimal solution of a certain $H_{\infty}$ norm for middle and high frequency ranges. The parameters of PID controller are obtained through solving some linear matrix inequalities (LMIs) with MATLAB. We find that the method is well applicable for middle frequency range of a system with different typical inputs and high frequency rang of a system with ramp input.

\section{PRELIMINARIES}

A. Approximate Model Matching

A standard feedback arrangement is shown in Fig.1.

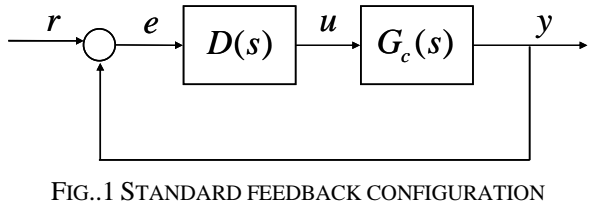

In this figure, $D(s)$ stands for the transfer function of controller in the complex plane and $G_{c}(s)$ stands for the transfer function of controlled objects. Then, according to the matching model approach, a reference model should be chosen firstly. Its open-loop transfer function can be denoted as $G_{r}(s)$. Thus the problem of controller design equates with attempting to derive $D(s)$ by sovling the inequality

$$
\left\|D(s) G_{c}(s)-G_{r}(s)\right\|_{\infty}<\varepsilon
$$

where $\varepsilon$ is a given positive real number. The smaller $\varepsilon$ is, the better model matching one can get [7].

\section{B. Generalization of KYP Lemma}

For a matrix $M$, its transpose and complex conjugate transpose are denoted by $M^{T}$ and $M^{*}$, respectively. For a Hermitian matrix $M, M>0$ and $M<0$ denote positive definiteness and negative definiteness, respectively. For matrices $\Phi$ and $P$, their Kronecker product is denoted by $\Phi \otimes P$.

Lemma 1 [4] Let complex matrices $A, B, \Pi=\Pi^{*}$, and $(\Phi, \Psi) \in \Omega$ be given where

$$
\begin{gathered}
\Omega:=\left\{(\Phi, \Psi) \mid \exists \alpha, \beta \in I R, M \in C^{2 \times 2} \text { such that } \Phi=M^{*}\left[\begin{array}{cc}
0 & \alpha \\
\alpha & 0
\end{array}\right] M,\right. \\
\left.\alpha \operatorname{det}(M) \neq 0, \Psi=M^{*}\left[\begin{array}{cc}
-1 & \beta \\
\beta & 1
\end{array}\right] M\right\} . \\
\text { Define } \Lambda:=\{\lambda \in C \mid \sigma(\lambda, \Phi)=0, \sigma(\lambda, \Psi) \geq 0\} \text {. Suppose }
\end{gathered}
$$
( $A, B$ ) is controllable and $A$ has no eigenvalues $\lambda$ such that $\sigma(\lambda, \Phi)=0$. Then the following statements are equivalent.

1) The following frequency domain condition 


$$
\left[\begin{array}{c}
(j w I-A)^{-1} B \\
I
\end{array}\right]^{*} \Pi\left[\begin{array}{c}
(j w I-A)^{-1} B \\
I
\end{array}\right] \leq 0
$$

holds for all $\lambda \in \Lambda$

2) There exist Hermitian matrices $P$ and $Q \geq 0$, such that

$$
\begin{gathered}
{\left[\begin{array}{cc}
A & B \\
I & 0
\end{array}\right]^{*} L(P, Q)\left[\begin{array}{cc}
A & B \\
I & 0
\end{array}\right]+\Pi \leq 0,} \\
L(P, Q):=\Phi \otimes P+\Psi \otimes Q .
\end{gathered}
$$

By choosing appropriate $\Phi$ and $\Psi$, the set $\Lambda$ can be specialized to define a certain range of the frequency variable $\lambda$. For the continuous-time setting

$$
\Phi=\left[\begin{array}{ll}
0 & 1 \\
1 & 0
\end{array}\right], \Lambda=\{j \omega: \omega \in F\}
$$

where $F$ is a subset of real numbers specified by an additional choice of $\Psi$, as shown in TABLE I.

TABLE I. CORRESPONDING RELATIONSHIP BETWEEN $F$ AND $\Psi$

\begin{tabular}{cccc}
\hline & LFR & MFR & HFR \\
\hline$F$ & $|\omega| \leq \varpi_{\ell}$ & $\varpi_{1} \leq \omega \leq \varpi_{2}$ & $|\omega| \geq \varpi_{h}$ \\
$\Psi$ & {$\left[\begin{array}{cc}-1 & 0 \\
0 & \varpi_{\ell}{ }^{2}\end{array}\right]$} & {$\left[\begin{array}{cc}-1 & j \varpi_{c} \\
-j \varpi_{c} & -\varpi_{1} \varpi_{2}\end{array}\right]$} & {$\left[\begin{array}{cc}1 & 0 \\
0 & -\varpi_{h}{ }^{2}\end{array}\right]$} \\
\hline
\end{tabular}

where $\varpi_{c}:=\left(\varpi_{1}+\varpi_{2}\right) / 2$.

\section{MAin Results}

We give out the main results in this section. Theorem 1 and Corollary 1 are suitable for middle frequency range. Theorem 2 and Corollary 2 are suitable for high frequency range.

Theorem 1: Let real matrices $A, B, C, D$ with appropriate dimensions, and real scalars $\varpi_{1} \leq \varpi_{2}$ be given. Suppose $(A, B)$ is controllable. Then the following statements are equivalent.

1) With $F:=\left\{\omega \in R: \operatorname{det}(j \omega I-A) \neq 0, \varpi_{1} \leq \omega \leq \varpi_{2}\right\}$ we have

$$
\left\|C(s I-A)^{-1} B+D\right\|_{\infty}^{F}<\varepsilon \quad \forall \omega \in F .
$$

2) There exist real symmetric matrices $P$ and $Q$ satisfying $\mathrm{Q}>0$ and

$$
\begin{aligned}
& {\left[\begin{array}{ll}
A & B \\
I & 0
\end{array}\right]^{T}\left[\begin{array}{cc}
-Q & P+j \varpi_{c} Q \\
P-j \varpi_{c} Q & -\varpi_{1} \varpi_{2} Q
\end{array}\right]\left[\begin{array}{cc}
A & B \\
I & 0
\end{array}\right]+} \\
& {\left[\begin{array}{cc}
C^{T} C & C^{T} D \\
D^{T} C & D^{T} D-\varepsilon^{2} I
\end{array}\right]<0}
\end{aligned}
$$

where $\varpi_{c}:=\left(\varpi_{1}+\varpi_{2}\right) / 2$.

Proof. According to the definition of $H_{\infty}$ norm of $G(s)=C(s I-A)^{-1} B+D$ and $\sigma_{\max }$ :

$$
\|G(s)\|_{\infty}=\sup _{\omega \in R} \sigma_{\max }(G(j \omega))
$$

where $\sigma_{\max }$ stands for the largest singular value [8]. $\|G(s)\|_{\infty}^{F}<\varepsilon$ equates with

$$
G^{*}(j w) G(j w)<\varepsilon^{2} I, \quad \forall \omega \in F .
$$

Since $G(j w)=C(j w I-A)^{-1} B+D$, we have

$\left(C(j w I-A)^{-1} B+D\right)^{*}\left(C(j w I-A)^{-1} B+D\right)-\varepsilon^{2} I<0 \quad \forall \omega \in F . \quad(8)$

Then for a real matrix $M$ with $M^{*}=M^{T}$, we obtain

$\left((j w I-A)^{-1} B\right)^{*} C^{T} C\left((j w I-A)^{-1} B\right)+\left((j w I-A)^{-1} B\right)^{*} C^{T} D+$

$D^{T} C\left((j w I-A)^{-1} B\right)+D^{T} D-\varepsilon^{2} I<0 \quad \forall \omega \in F$

It can be written in the form of multiplication of matrices

$$
\left[\begin{array}{c}
(j w I-A)^{-1} B \\
I
\end{array}\right]\left[\begin{array}{cc}
C^{T} C & C^{T} D \\
D^{T} C & D^{T} D-\varepsilon^{2} I
\end{array}\right]\left[\begin{array}{c}
(j w I-A)^{-1} B \\
I
\end{array}\right]<0 \quad \forall \omega \in F(10)
$$

By Lemma 1, it is equivelent with the statement: there exist $P$ and $Q$ satisfying $Q>0$, and

$$
\begin{aligned}
& {\left[\begin{array}{ll}
A & B \\
I & 0
\end{array}\right]^{T}\left[\begin{array}{cc}
-Q & P+j \varpi_{c} Q \\
P-j \varpi_{c} Q & -\varpi_{1} \varpi_{2} Q
\end{array}\right]\left[\begin{array}{cc}
A & B \\
I & 0
\end{array}\right]+} \\
& {\left[\begin{array}{cc}
C^{T} C & C^{T} D \\
D^{T} C & D^{T} D-\varepsilon^{2} I
\end{array}\right]<0 .}
\end{aligned}
$$

The proof is completed.

The dual version of Theorem 1 is stated as follows.

Corollary 1: Let real matrices $A, B, C, D$ with appropriate dimensions, and real scalars $\varpi_{1} \leq \varpi_{2}$ be given. Then the following statements are equivalent.

1) With $F:=\left\{\omega \in R: \operatorname{det}(j \omega I-A) \neq 0, \varpi_{1} \leq \omega \leq \varpi_{2}\right\}$ we have

$$
\left\|C(s I-A)^{-1} B+D\right\|_{\infty}^{F}<\varepsilon \quad \forall \omega \in F .
$$

2) There exist real symmetric matrices $P$ and $Q$ satisfying $Q>0$ and

$$
\begin{aligned}
& {\left[\begin{array}{ll}
A & I \\
C & 0
\end{array}\right]\left[\begin{array}{cc}
-Q & P-j \varpi_{c} Q \\
P+j \varpi_{c} Q & -\varpi_{1} \varpi_{2} Q
\end{array}\right]\left[\begin{array}{ll}
A & I \\
C & 0
\end{array}\right]^{T}+} \\
& {\left[\begin{array}{cc}
B B^{T} & B D^{T} \\
D B^{T} & D D^{T}-\varepsilon^{2} I
\end{array}\right]<0}
\end{aligned}
$$

where $\varpi_{c}:=\left(\varpi_{1}+\varpi_{2}\right) / 2$.

Theorem 2: Let real matrices $A, B, C, D$ with appropriate dimensions be given. Suppose $(A, B)$ is controllable. Then the following statements are equivalent.

1) With $F:=\left\{\omega \in R: \operatorname{det}(j \omega I-A) \neq 0, \varpi_{h} \leq|\omega|\right\}$ we have

$$
\left\|C(s I-A)^{-1} B+D\right\|_{\infty}^{F}<\varepsilon \quad \forall \omega \in F .
$$

2) There exist real symmetric matrices $P$ and $Q$ satisfying $Q>0$ and

$$
\left[\begin{array}{cc}
A & B \\
I & 0
\end{array}\right]^{T}\left[\begin{array}{cc}
Q & P \\
P & -\bar{\omega}_{h}^{2} Q
\end{array}\right]\left[\begin{array}{cc}
A & B \\
I & 0
\end{array}\right]+
$$




$$
\left[\begin{array}{cc}
C^{T} C & C^{T} D \\
D^{T} C & D^{T} D-\varepsilon^{2} I
\end{array}\right]<0
$$

Theorem 3: Let real matrices $A, B$ and $C$ with appropriate dimensions, and real scalars $\varpi_{1} \leq \varpi_{2}$ be given. Then the following statements are equivalent.

1) With $F:=\left\{\omega \in R: \operatorname{det}(j \omega I-A) \neq 0, \varpi_{1} \leq \omega \leq \varpi_{2}\right\}$ we have

$$
\left\|C(s I-A)^{-1} B\right\|_{\infty}^{F}<\varepsilon \quad \forall \omega \in F
$$

2) There exist real symmetric matrices $P$ and $Q$ satisfying $\mathrm{Q}>0$ and

$$
\left[\begin{array}{ccc}
N & -A Q C^{r}+P C^{r}+j \omega_{-} Q C^{r} & B \\
-C Q A^{r}+C P-j \sigma_{c} C Q & -C Q C^{r}-\varepsilon^{2} I & 0 \\
B^{r} & 0 & -I
\end{array}\right]<0
$$

where

$N:=-A Q A^{T}+P A^{T}+j \varpi_{c} A^{T}+A P-j \varpi_{c} A Q-\varpi_{1} \varpi_{2} Q$.

Proof. By Corollary 1, $\left\|C(s I-A)^{-1} B\right\|_{\infty}^{F}<\varepsilon$ if and only if there exist real symmetric matrices $P$ and $Q$ satisfying $Q>0$ and

$$
\begin{gathered}
{\left[\begin{array}{ll}
A & I \\
C & 0
\end{array}\right]^{T}\left[\begin{array}{cc}
-Q & P-j \varpi_{c} Q \\
P+j \varpi_{c} Q & -\varpi_{1} \varpi_{2} Q
\end{array}\right]} \\
{\left[\begin{array}{ll}
A & I \\
C & 0
\end{array}\right]\left[\begin{array}{cc}
B B^{T} & 0 \\
0 & -\varepsilon^{2} I
\end{array}\right]<0}
\end{gathered}
$$

It is then further equivalent to

$$
\begin{gathered}
{\left[\begin{array}{cc}
A & I \\
C & 0
\end{array}\right]\left[\begin{array}{cr}
-Q & P-j \varpi_{c} Q \\
P+j \varpi_{c} Q & -\varpi_{1} \varpi_{2} Q
\end{array}\right]\left[\begin{array}{cc}
A & I \\
C & 0
\end{array}\right]^{T}} \\
+\left[\begin{array}{cc}
0 & 0 \\
0 & -\varepsilon^{2} I
\end{array}\right]+\left[\begin{array}{l}
B \\
0
\end{array}\right] I\left[\begin{array}{ll}
B^{T} & 0
\end{array}\right]<0
\end{gathered}
$$

Since

$$
\begin{gathered}
{\left[\begin{array}{cc}
A & I \\
C & 0
\end{array}\right]\left[\begin{array}{cr}
-Q & P-j \varpi_{c} Q \\
P+j \varpi_{c} Q & -\varpi_{1} \varpi_{2} Q
\end{array}\right]\left[\begin{array}{cc}
A & I \\
C & 0
\end{array}\right]^{T}+\left[\begin{array}{ll}
0 & 0 \\
0 & -\varepsilon^{2} I
\end{array}\right]} \\
=\left[\begin{array}{cc}
\Omega_{1} & -A Q C^{T}+P C^{T}+j \varpi_{c} Q C^{T} \\
-C Q A^{T}+C P-j \varpi_{c} C Q & -C Q C^{T}-\varepsilon^{2} I
\end{array}\right]
\end{gathered}
$$

where $\Omega_{1}=-A Q A^{T}+P A^{T}+j \varpi_{c} A^{T}+A P-j \varpi_{c} A Q-\varpi_{1} \varpi_{2} Q$, we can simply obtain the final result by the Schur lemma.

Theorem 4: Let real matrices $A, B$ and $C$ with appropriate dimensions be given. Suppose $(A, B)$ is controllalbe. Then the following statements are equivalent.

1) With $F:=\left\{\omega \in R: \operatorname{det}(j \omega I-A) \neq 0, \varpi_{h} \leq|\omega|\right\}$ we have

$$
\left\|C(s I-A)^{-1} B\right\|_{\infty}^{F}<\varepsilon \quad \forall \omega \in F .
$$

2) There exist real symmetric matrices $P$ and $Q$ satisfying $Q>0$ and

$$
\left[\begin{array}{ccc}
A Q A^{T}+P A^{T}+A P-\varpi_{h}^{2} Q & A Q C^{T}+P C^{T} & B \\
C Q A^{T}+C P & C Q C^{T}-\varepsilon^{2} I & 0 \\
B^{T} & 0 & -I
\end{array}\right]<0 .
$$

\section{NUMERICAL SIMULATIONS}

\section{A. The Plant}

Consider a controlled object with the transfer function $G_{c}(s)=C_{c}\left(s I-A_{c}\right)^{-1} B_{c}$, where

$$
\begin{gathered}
A_{c}=\left[\begin{array}{ccc}
0 & 1 & 0 \\
0 & 0 & 1 \\
-1 & -4 & -2
\end{array}\right], B_{c}=\left[\begin{array}{l}
0 \\
0 \\
1
\end{array}\right] \\
C_{c}=\left[\begin{array}{lll}
1 & 3 & 0
\end{array}\right], D_{D}=0
\end{gathered}
$$

The aim is to design a PID controller such that there is no static error for middle frequency range with step signal inputs and high frequency range with ramp signal inputs. Additionally, the overshoot $\sigma$ has to be no more than $10 \%$. The rise time $t_{r}$ and regulation time $t_{s}$ are both asked to be no more than $1 \mathrm{~s}$.

B. Design of PID controller

The controller is given by

$$
D(s)=K_{P}+\frac{K_{I}}{s}+\frac{K_{D} s}{T_{D} s+1}=C_{D}\left(s I-A_{D}\right)^{-1} B_{D}+D_{D}
$$

where

$$
\begin{gathered}
A_{D}=\left[\begin{array}{cc}
0 & 0 \\
1 & \frac{-1}{T_{D}}
\end{array}\right], B_{D}=\left[\begin{array}{c}
\frac{K_{I}}{T_{D}} \\
K_{I}-\frac{K_{D}}{T_{D}}
\end{array}\right] \\
C_{D}=\left[\begin{array}{ll}
0 & 1
\end{array}\right], D_{D}=K_{P}+K_{D} / T_{D} .
\end{gathered}
$$

The transfer function of reference model is

$$
G_{r}(s)=C_{r}\left(s I-A_{r}\right)^{-1} B_{r} \text {. }
$$

Denote $G(s)=D(s) G_{c}(s)-G_{r}(s)=C(s I-A)^{-1} B$. Then the matrices $A, B$ and $C$ can be derived

$$
A=\left[\begin{array}{ccc}
A_{C} & B_{C} C_{D} & 0 \\
0 & A_{D} & 0 \\
0 & 0 & A_{r}
\end{array}\right], B=\left[\begin{array}{c}
B_{C} D_{D} \\
B_{D} \\
B_{r}
\end{array}\right], C=\left[\begin{array}{lll}
C_{C} & 0 & -C_{r}
\end{array}\right] .
$$

The problem of optimization design of PID controller for middle and high frequency ranges is converted into computing the parameters of controller through $\|G(s)\|_{\infty}^{F}<\varepsilon$, where $F:=\left\{\omega \in R: \operatorname{det}(j \omega I-A) \neq 0, \varpi_{h} \leq|\omega|\right\} \quad$ or

$$
F:=\left\{\omega \in R: \operatorname{det}(j \omega I-A) \neq 0, \varpi_{1} \leq \omega \leq \varpi_{2}\right\} .
$$

According to the request of the performance index, we can choose the reference model as $G_{r}(s)=36 /\left(s^{2}+6 \sqrt{2} s\right)$. 
Then, we can compute the practical parameters of the PID controllers for the middle frequency rang (MFR) and high frequency rang (HFR).

1) PID controller designed for MFR . Given that $F:=\{\omega \in R: 1.32 \leq \omega \leq 8.22\}, T_{D}=0.05 \mathrm{~s}$, and the error limit $\varepsilon=\sqrt{0.05}$, we can obtain by solving LMI (20) $K_{P}=2.5, K_{I}=3.8, K_{D}=1.0$.

Therefore, the step response curve is shown in Fig.2, and the bode graphs are shown in Fig.3. As is seen, the closedloop control system is stable.

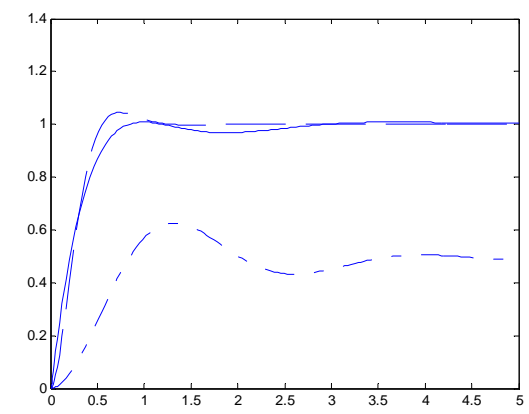

Fig.2 STEP RESPONSE CURVES

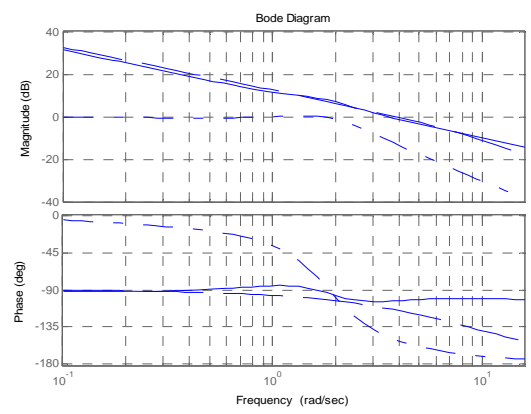

FIG.3 BODE GRAPH

The solid line stands for the response curve of the system with PID controller. The dot dash line stands for the response curve of the original system without controller, and the dashed line stands for that of reference model.

2) PID controller designed for HFR. Similarly, when the input is a ramp signal and $F:=\{\omega \in R: 16.5 \leq|\omega|\}$, we can design a PID controller for high frequency range of the system. Similarly, we can obtain that $K_{P}=12, K_{I}=4$, $K_{D}=0.001$. Therefore, the corresponding ramp response curve is shown in Fig.4, and the bode graphs are shown in Fig.5. As is seen, the closed-loop control system is also stable.

\section{CONCLUSION}

This paper has proposed a method of designing PID controller for middle and high frequency ranges. Since the design of PID controller for low frequency range has been studied in [5], we only focus on PID controller design in middle and high frequency ranges. Simulation results has illustrated the effectiveness of the proposed method. However, how to switch the parameters of the PID controller for different frequency ranges still needs further research.

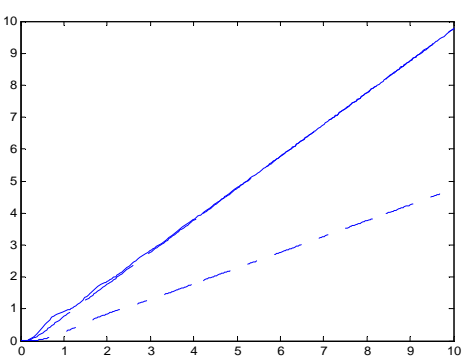

FIG.4 RAMP RESPONSE CURVES

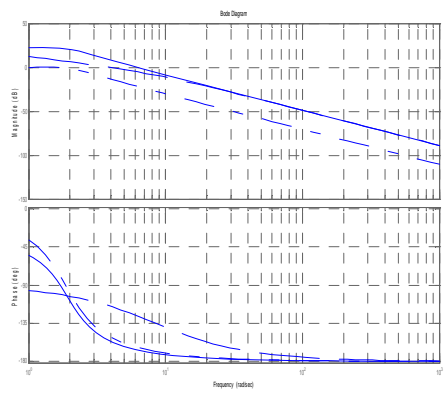

FIG.5 BODE GRAPH

\section{REFERENCES}

[1] G. Zames, "Feedback and optimal sensitivity: model reference transformations, multiplicative seminorms and approximation inverses,” IEEE Trans on Automatic Control, 1981, 26(2): 301-320.

[2] J. C. Doyle, K. Glover, P. Khargonekar, et al. "State-space solutions to standard $\mathrm{H}_{2}$ and $H_{\infty}$ control problems," IEEE Trans on Automatic Control, 1989, 34(8): 831-847.

[3] T. Iwasaki, S. Hara, "Generalized KYP lemma: Unified frequency domain inequalities with design applications," IEEE Trans on Automatic Control, 2005, 50(1):41-59.

[4] T. Iwasaki, S.Hara, "Generalization of Kalman-Yakubovic-Popov lemma for restricted frequency inequalities," Proc. of American Control Conference 2003, Denver, Colorado, June 4-6, 2003, pp. 3828-3833.

[5] G. Ma, Q. Chen, W. Hu, "Optimal design of PID controller based on window $H_{\infty}$ norm," Acta Automatica Sinica, 2007, 33(9):1000-1003.

[6] A. Dehghani, A. Lanzon, B. D. O. Anderson, "A two-degree-offreedom H-infinity control design method for robust model matching ," Int J of Robust and Nonlinear Control, 2006,16(10): 467483.

[7] A. Dehghani, A. Lanzon, B. D. O. Anderson, “An $H$ model referencing design utilizing a two degree of freedom controller scheme," The 44th IEEE Conference on Decision and Control and. European Control Conference ECC 2005. Plaza de España Seville, (Spain), December 12-15, 2005, pp. 2302-2307.

[8] G. Ma, D. Wang, "Analysis and design of linear control systems in window frequency domain,” Control and Decision, 2008, 23(9):10211024. 